Revue internationale de l'économie sociale

Recma

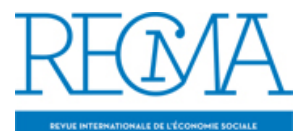

\title{
Les coopératives au Maroc : enjeux et évolutions
}

\section{Saïd Ahrouch}

Numéro 322, octobre 2011

URI : https://id.erudit.org/iderudit/1020728ar

DOI : https://doi.org/10.7202/1020728ar

Aller au sommaire du numéro

\section{Éditeur(s)}

Association Recma

\section{ISSN}

1626-1682 (imprimé)

2261-2599 (numérique)

Découvrir la revue

\section{Citer cet article}

Ahrouch, S. (2011). Les coopératives au Maroc : enjeux et évolutions. Revue internationale de l'économie sociale, (322), 23-26.

https://doi.org/10.7202/1020728ar d'utilisation que vous pouvez consulter en ligne.

https://apropos.erudit.org/fr/usagers/politique-dutilisation/ 


\title{
LES COOPÉRATIVES AU MAROC: ENJEUX ET ÉVOLUTIONS
}

\author{
par Saïd Ahrouch*
}

* Université lbn Zohr, Agadir.

(1) Ce texte est issu d'une communication présentée au colloque européen de I'ACl "Les contributions des coopératives à une économie plurielle", organisé à I'université Lumière-Lyon 2 les 2, 3 et 4 septembre 2010.

A travers leurs valeurs de démocratie, de solidarité, de partage et d'entraide, les coopératives jouent un rôle de plus en plus important dans le développement économique et social du Maroc. Leur attractivité croît surtout depuis 2005, année du lancement de l'Initiative nationale du développement humain (INDH) encourageant la création et la pérennisation des structures de l'économie sociale et solidaire. La présente contribution traite des enjeux de l'entrepreneuriat coopératif, examine les appuis institutionnel et fiscal offerts par l'Etat marocain aux coopératives et présente enfin le poids économique et social des coopératives dans l'économie marocaine ${ }^{(1)}$.

\section{L'appui institutionnel}

(2) Article 6 de la loi 24-83 fixant le statut général des coopératives et les missions de l'Office du développement de la coopération au Maroc.
Dès l'indépendance du Maroc, en 1956, le modèle économique coopératif a constitué un choix stratégique pour ce pays, afin d'assurer une mobilisation nationale pour la modernisation et le développement des secteurs traditionnels, notamment l'agriculture, qui bénéficie encore aujourd'hui d'une exonération fiscale. L'encouragement à l'organisation de production sous forme coopérative traduisait à l'époque le rôle que peuvent jouer les coopératives dans le progrès national, l'amélioration de la situation économique et sociale et l'épanouissement personnel des coopérateurs. Au niveau institutionnel, a été créé en 1963 l'Office de développement de la coopération (ODCO; www.odco.gov.ma), comme structure administrative chargée de l'accompagnement des coopératives dans les domaines de la formation et de l'information et comme appui juridique. Restructuré en 1975, cet office est devenu une entreprise publique jouissant de la personnalité morale et d'autonomie financière et administrative. Actuellement, les missions de l'ODCO sont fixées par la loi 24-83 (art. 77). Il s'agit principalement, sauf en ce qui concerne les coopératives de la réforme agraire, de soutenir les coopératives et leurs unions aussi bien à la création (instruction et centralisation des demandes) qu'en cours d'activité (formation, information, assistance juridique, œuvres sociales, mise à niveau et restructuration...). Les coopératives ne peuvent traiter qu'avec leurs membres. Ce principe d'exclusivisme ${ }^{(2)}$ 
pouvant limiter leur développement, certaines coopératives de production et de commercialisation peuvent bénéficier d'une dérogation administrative temporaire.

De même, selon la loi marocaine (article 30 de la loi 24-83), les statuts de la coopérative peuvent prévoir une rémunération du capital, mais le taux d'intérêt ne doit pas être supérieur à $6 \%$ et les intérêts ne peuvent être servis que si des excédents ont été réalisés au cours de l'exercice. Ce point statutaire, ajouté à celui de la non-répartition des excédents mis en réserve (art. 2 de la loi 24-83), sollicite encore l'attention des coopérateurs dans le but d'introduire certains aménagements juridiques aux statuts coopératifs.

Cet appui institutionnel n'a toutefois pas été accompagné par de vraies politiques gouvernementales relatives à l'économie sociale et solidaire en général et aux coopératives en particulier. Ce n'est qu'à partir du lancement de l'Initiative nationale de développement humain, en mai 2005 (voir "Temps forts", Recma, n³15, NDLR), que les choix publics en matière sociale ont pris de l'ampleur, avec plus de clarification, de programmation et de ressources financières. Cette évolution, salutaire pour les coopératives, n'en a pas moins été paradoxale du point de vue fiscal.

\section{L'appui fiscal}

(3) Bensalem Fennassi, 2004, "Coopératives: la fin des privilèges ", Aujourd'hui le Maroc, $n^{\circ} 739,29$ avril.
En matière fiscale, les coopératives bénéficient, au Maroc, d'exonérations importantes. Il s'agit principalement des exonérations de l'impôt des patentes, de la taxe urbaine, de l'impôt sur les bénéfices professionnels (article 87 de la loi 24-83) et de la taxe sur la vente de produits et sur les opérations et services effectués pour le compte des adhérents (article 88 de la loi 24-83). Toutefois, si les coopératives étaient historiquement exonérées sans limitation en vertu de leurs statuts, ce n'est plus le cas actuellement. Depuis 2005, en effet, les pouvoirs publics ont soumis à l'impôt sur les sociétés (IS) et à la taxe sur la valeur ajoutée (TVA) les coopératives réalisant un chiffre d'affaires supérieur à 1 million de dirhams (environ 90000 euros, NDLR).

Selon le gouvernement marocain, cette imposition visait à rétablir la concurrence loyale dans plusieurs branches d'activité. Certaines coopératives agricoles, et notamment laitières, ont pu en effet développer des structures capitalistes un peu éloignées des petits éleveurs ${ }^{(3)}$. De leur côté, les représentants des coopératives accusent le gouvernement d'avoir cédé aux pressions exercées par des opérateurs privés organisés en sociétés commerciales, qui n'ont cessé de critiquer les situations de concurrence déloyale.

Depuis, pour défendre leurs intérêts, les coopératives ont décidé de réagir en commun par la création de la Fédération nationale des coopératives. L'objectif est de convaincre le gouvernement d'abroger les dispositions 
(4) Coopérative agricole créée en 1987: 112 adhérents particuliers, 70 coopératives adhérentes (dont 14000 éleveurs); capital social, 140 millions de dirhams $(12,5$ millions d'euros); chiffre d'affaires, 1,7 milliard de dirhams $(152$ millions d'euros); emplois directs, 3300 personnes. au terme desquelles elles sont imposées. Cette imposition, selon le président de l'Union des coopératives agricoles marocaines (Uncam), allait induire le licenciement d'au moins la moitié des effectifs employés par les coopératives. Toutefois, ces licenciements n'ont jusqu'à présent pas eu lieu, au moins à la Copag ${ }^{(4)}$, la plus grande coopérative concernée par ces mesures.

\section{L'évolution des coopératives au Maroc de 2005 à 2009}

Il est difficile de trouver des statistiques fiables sur les coopératives pour mener une analyse approfondie et tirer des conclusions significatives, car ces données sont éparpillées, insuffisantes et parfois absentes pour certaines périodes. Cela étant, le lancement de l'INDH en 2005 a donné un coup de pouce à la création des coopératives, notamment dans le milieu rural, qui souffrait de l'exclusion et de la pauvreté. Ainsi, de 2005-2009 le nombre de coopératives a crû de 38,3\% (tableau 1). Cette évolution traduit le rôle déterminant du tissu coopératif dans le développement économique et social au Maroc, grâce à une volonté politique réelle.

Quant à la répartition sectorielle (tableau 2), l'agriculture est le secteur le plus important. Il occupe le premier rang (62\%) dans le tissu coopératif, comme il l'occupe dans la participation au PIB. Il est suivi respectivement par les secteurs de l'habitat et de l'artisanat. Notons aussi que la structuration des activités liées à l'huile d'argan a donné naissance à plusieurs coopératives dans ce domaine qui représente aujourd'hui 2,3\% du tissu coopératif national. Une place d'autant plus notable que ces coopératives

\section{Tableau 1}

L'évolution de l'effectif des coopératives au Maroc de 2005 à 2009

\begin{tabular}{|l|r|r|r|r|r|}
\hline Année & 2005* $^{*}$ & 2006* $^{*}$ & 2007* $^{*}$ & 2008* $^{*}$ & 2009*$^{*}$ \\
\hline Nombre de coopératives & 4985 & 5276 & 5749 & 6286 & 6895 \\
\hline Taux de croissance en \% & 3,3 & 5,9 & 9 & 9,35 & 9,7 \\
\hline
\end{tabular}

*Attaaoun, $n^{\circ} 88$, hiver 2009, p. 9

** www.odco.gov.ma (rubrique "Statistiques").

\section{Tableau 2}

La répartition sectorielle des coopératives (données de 2008)

\begin{tabular}{|l|c|c|c|c|}
\hline Secteur & Agriculture & Habitat & Artisanat & Autres secteurs \\
\hline Pourcentage & 62,1 & 15,4 & 12,3 & 8,2 \\
\hline
\end{tabular}

Sources: Attaaoun, $n^{\circ}$ 91, automne 2009, p. 25 (publication de l'ODCO). 
sont à $95 \%$ féminines. En fait, l'entrepreneuriat féminin en coopératives constitue un tournant important dans la société marocaine, permettant aux femmes d'avoir l'autonomie financière et plus de pouvoir dans la prise de décision.

Le secteur coopératif enregistre ainsi chaque mois la création de nouvelles coopératives dans différents secteurs d'activité. A titre d'exemple, au cours du mois de novembre 2010, 106 coopératives se sont créées, regroupant 2167900 dirhams (194000 euros) de capital, 1451 adhérents et 1290 emplois. Ces nouvelles structures envisagent d'investir 8671600 dirhams (775000 euros) pendant les quatre prochaines années, dans la perspective de créer 5804 postes supplémentaires. Il est particulièrement important de relever que, parmi ces 106 nouvelles entités, 14 sont des coopératives de femmes (136 coopératrices). Le poids du secteur coopératif s'accroît: 347684 adhérents et 22502 employés en 2006 (Attaaoun, n 91, automne 2009), 365255 adhérents en 2010. Dans la même période, son capital a crû de quelque 33 millions de dirhams (soit environ 3 millions d'euros) pour atteindre 6 milliards de dirhams (540 millions d'euros) au 30 juin 2010.

Au Maroc l'enjeu est d'ampleur, étant donné l'immensité des domaines de coopération et les objectifs de développement économique, social et humain. L'initiative nationale de développement humain, dans son axe d'activités génératrices de revenus, encourage et soutient le modèle coopératif, surtout celui des femmes en milieu rural. En plus de l'INDH, le plan Maroc vert constitue une plateforme importante de développement des coopératives agricoles. 